\title{
An accurate allowance for initial and final state interactions in the treatment of the alpha-alpha Bremsstrahlung
}

Adam M. Arslanaliev ${ }^{1,2 \star}$ and Aleksandr V. Shebeko ${ }^{2}$

1 V. N. Karazin National University, Kharkiv, Ukraine

2 Institute for Theoretical Physics, National Research Center Kharkiv

Institute of Physics \& Technology, Kharkiv, Ukraine

$\star$ arslanaliev.kh@gmail.com

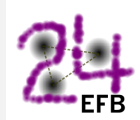

Proceedings for the 24th edition of European Few Body Conference,

Surrey, UK, 2-6 September 2019

doi:10.21468/SciPostPhysProc.3

\begin{abstract}
The alpha-alpha bremsstrahlung is studied using the generalization of the Siegert theorem. The corresponding amplitude is written in the gauge invariant form. Special attention is paid to taking into account the Coulomb interaction. Some correlation function is found and its dependence on the strong alpha-alpha interaction is discussed.
\end{abstract}

(c) (1) Copyright A. M. Arslanaliev and A. V. Shebeko. This work is licensed under the Creative Commons Attribution 4.0 International License.

Published by the SciPost Foundation.
Received 13-10-2019

Accepted 29-11-2019

Published 27-02-2020

doi:10.21468/SciPostPhysProc.3.042

\section{Theoretical background}

Our departure point in describing electromagnetic (EM) interactions with nuclei (in general, bound systems of charged particles) is to rely upon the Fock-Weyl criterion and the generalization of the Siegert theorem (see [1] for details and [2,3], where this approach is compared to that by Friar and Fallieros $[4,5])$.

The properties of gauge invariance for a quantum-mechanical system that interacts with an EM field can be formulated by considering the Schrödinger equation

$$
\imath \frac{\partial \Psi}{\partial t}=H_{\text {total }}\left\{A_{\mu}\right\} \Psi
$$

and the gradient displacement

$$
A_{\mu}(\mathbf{x}, t) \rightarrow A_{\mu}^{\prime}(\mathbf{x}, t)=A_{\mu}(\mathbf{x}, t)+\partial_{\mu} G(\mathbf{x}, t)
$$

of the EM potential $A^{\mu}(\mathbf{x}, t)=\left(A^{0}(\mathbf{x}, t), \mathbf{A}(\mathbf{x}, t)\right)$ at the space-time point $x=(\mathbf{x}, t)$ with an arbitrary function $G(x)$. The theory is gauge invariant if there exists a unitary transformation $\Psi \rightarrow \Psi^{\prime}=U \Psi$ after Fock such that Eq. (1) remains unchanged in its form viz.,

$$
\imath \frac{\partial \Psi^{\prime}}{\partial t}=H_{\text {total }}\left\{A_{\mu}^{\prime}\right\} \Psi^{\prime} \rightarrow H_{\text {total }}\left\{A_{\mu}^{\prime}\right\}=U H_{\text {total }}\left\{A_{\mu}\right\} U^{\dagger}+\imath \frac{\partial U}{\partial t} U^{\dagger} .
$$


In the first order in charge $e$ it gives the continuity equation (CE) for the current operator $J^{\mu}(\mathbf{x})=(\rho(\mathbf{x}), \mathbf{J}(\mathbf{x}))$ :

$$
\imath \operatorname{div} \mathbf{J}(\mathbf{x})=[H, \rho(\mathbf{x})] \rightarrow[\boldsymbol{P}, \boldsymbol{J}(0)]=[H, \rho(0)],
$$

where $H$ is the nuclear part of $H_{\text {total }}\left\{A_{\mu}\right\}$ and we take into account the property of translational invariance

$$
\exp (-\imath \mathbf{P} \cdot \mathbf{a}) \mathbf{J}(\mathbf{x}) \exp (\iota \mathbf{P} \cdot \mathbf{a})=\mathbf{J}(\mathbf{x}+\mathbf{a})
$$

with arbitrary displacement $\mathbf{a}, \mathbf{P}$ is the total momentum operator for the nucleus (bound system).

According to refs. $[2,3]$, the amplitude of the reaction $A+B \rightarrow A^{\prime}+B^{\prime}+\gamma$ for the photon emission with energy $E_{\gamma}$, momentum $\mathbf{k}_{\gamma}$ and polarization $\varepsilon^{\mu}=\left(\varepsilon^{0}, \boldsymbol{\varepsilon}\right)$

$$
T_{i f}=\left[2(2 \pi)^{3} E_{\gamma}\right]^{-1 / 2}\left\langle\mathbf{P}_{i}-\mathbf{k}_{\gamma} ; f\left|\varepsilon^{\mu} \hat{J}_{\mu}(0)\right| \mathbf{P}_{i} ; i\right\rangle
$$

can be expressed through electric $\mathbf{E}\left(\mathbf{k}_{\gamma}\right)$ and magnetic $\mathbf{H}\left(\mathbf{k}_{\gamma}\right)$ field strengths

$$
\mathbf{E}\left(\mathbf{k}_{\gamma}\right)=i\left[2(2 \pi)^{3} E_{\gamma}\right]^{-1 / 2}\left(E_{\gamma} \boldsymbol{\varepsilon}\left(\mathbf{k}_{\gamma}\right)-\mathbf{k}_{\gamma} \varepsilon_{0}\left(\mathbf{k}_{\gamma}\right)\right), \quad \mathbf{H}\left(\mathbf{k}_{\gamma}\right)=i\left[2(2 \pi)^{3} E_{\gamma}\right]^{-1 / 2} \mathbf{k}_{\gamma} \times \boldsymbol{\varepsilon}\left(\mathbf{k}_{\gamma}\right),
$$

and matrix elements $\mathbf{D}_{i f}\left(\mathbf{k}_{\gamma}\right)$ and $\mathbf{M}_{i f}\left(\mathbf{k}_{\gamma}\right)$ of the so-called generalized electric and magnetic dipole moments of nucleus (bound system)

$$
T_{i f}=\mathbf{E}\left(\mathbf{k}_{\gamma}\right) \mathbf{D}_{i f}\left(\mathbf{k}_{\gamma}\right)+\mathbf{H}\left(\mathbf{k}_{\gamma}\right) \mathbf{M}_{i f}\left(\mathbf{k}_{\gamma}\right) .
$$

These formulae were derived without separation of the center-of-mass (CM) motion, and thus they can be used in relativistic nuclear models (see, e.g., [6]).

\section{Nonrelativistic consideration}

We will confine ourselves to nonrelativistic approach in which the nuclear Hamiltonian

$$
H=\frac{\mathbf{P}^{2}}{2 M}+H_{\text {int }} \equiv K_{C M}+H_{\text {int }}
$$

is divided into the kinetic energy operator $K_{C M}$ of the center-of-mass (CM) motion, where $M$ is the total mass of nuclear system and the intrinsic Hamiltonian $H_{\text {int }}$ depends on internal variables of interacting nucleons. For a nonrelativistic system the amplitude (8) is given by (details in [3]):

$$
\begin{gathered}
T_{i f}=\left\langle f\left|T_{i n t}\right| i\right\rangle, \quad T_{i n t}=\mathbf{E}\left(\mathbf{k}_{\gamma}\right) \mathbf{D}\left(\mathbf{k}_{\gamma}\right)+\mathbf{H}\left(\mathbf{k}_{\gamma}\right) \mathbf{M}\left(\mathbf{k}_{\gamma}\right), \\
\mathbf{D}\left(\mathbf{k}_{\gamma}\right)=\frac{1}{E_{\gamma}} \int_{0}^{1}\left(\mathbf{P}_{i}-\lambda \mathbf{k}_{\gamma}|\mathbf{R}[H, \rho(0)]| \mathbf{P}_{i}\right) d \lambda, \quad \mathbf{M}\left(\mathbf{k}_{\gamma}\right)=-\int_{0}^{1}\left(\mathbf{P}_{i}-\lambda \mathbf{k}_{\gamma}|\mathbf{R} \times \mathbf{J}(0)| \mathbf{P}_{i}\right) \lambda d \lambda .
\end{gathered}
$$

In this context let us recall that the $H$ eigenstates can be factorized as $\left.\left|\mathbf{P}_{i} ; i\right\rangle=\mid \mathbf{P}_{i}\right)|i\rangle$ $\left.\left|\mathbf{P}_{i}-\mathbf{k}_{\gamma} ; f\right\rangle=\mid \mathbf{P}_{i}-\mathbf{k}_{\gamma}\right)|f\rangle$, where the bracket $\left.\mid\right)$ is used to represent a vector in the space of the $\mathrm{CM}$ coordinate $\mathbf{R}$ so $\hat{\mathbf{P}} \mid \mathbf{P})=\mathbf{P} \mid \mathbf{P})$ and |\rangle a vector in the internal space, so $H_{\text {int }}|i(f)\rangle=E_{i(f)}^{\text {int }}|i(f)\rangle$. Further, with help of the relation $[H, \mathbf{R}]=-i \mathbf{P} / M$ we find

$(2 \pi)^{3} \mathbf{D}\left(\mathbf{k}_{\gamma}\right)=\frac{1}{E_{\gamma}} \int_{0}^{1} d \lambda\left\{\left[\mathbf{D}_{\text {int }}\left(\lambda \mathbf{k}_{\gamma}\right), H_{\text {int }}\right]+\lambda \frac{\mathbf{k}_{\gamma} \cdot\left(2 \mathbf{P}_{i}-\lambda \mathbf{k}_{\gamma}\right)}{2 M} \mathbf{D}_{\text {int }}\left(\lambda \mathbf{k}_{\gamma}\right)+i \frac{\mathbf{P}_{i}-\lambda \mathbf{k}_{\gamma}}{M} \rho_{\text {int }}\left(\lambda \mathbf{k}_{\gamma}\right)\right\}$, 


$$
\begin{gathered}
\rho_{\text {int }}\left(\lambda \mathbf{k}_{\gamma}\right)=(2 \pi)^{3}\left(\mathbf{P}_{i}-\lambda \mathbf{k}_{\gamma}|\rho(0)| \mathbf{P}_{i}\right), \\
\mathbf{D}_{\text {int }}\left(\lambda \mathbf{k}_{\gamma}\right)=-(2 \pi)^{3}\left(\mathbf{P}_{i}-\lambda \mathbf{k}_{\gamma}|\mathbf{R} \rho(0)| \mathbf{P}_{i}\right)=\frac{i}{\lambda} \nabla_{\mathbf{k}_{\gamma}} \rho_{\text {int }}\left(\lambda \mathbf{k}_{\gamma}\right) .
\end{gathered}
$$

For the one-body charge-density operator:

$$
\rho^{[1]}(\mathbf{x})=\sum_{\alpha} \rho_{\alpha}(\mathbf{x}), \rho_{\alpha}(\mathbf{x})=\rho_{p}\left(\mathbf{x}-\mathbf{r}_{\alpha}\right) \pi_{p}(\alpha)+\rho_{n}\left(\mathbf{x}-\mathbf{r}_{\alpha}\right) \pi_{n}(\alpha),
$$

we get the Fourier transforms for

the charge density $\rho_{\text {int }}\left(\mathbf{k}_{\gamma}\right)=\left.\int_{0}^{1} d \lambda \sum_{i=1}^{8} f_{i}^{c h}(\mathbf{q}) \exp \left(-i \mathbf{q}\left(\mathbf{r}_{i}-\mathbf{R}\right)\right)\right|_{\mathbf{q}=\lambda \mathbf{k}_{\gamma}}$

the electric dipole moment $\quad \mathbf{D}_{\text {int }}\left(\mathbf{k}_{\gamma}\right)=\left.i \nabla_{\mathbf{q}} \int_{0}^{1} d \lambda \sum_{i=1}^{8} f_{i}^{c h}(\mathbf{q}) \exp \left(-i \mathbf{q}\left(\mathbf{r}_{i}-\mathbf{R}\right)\right)\right|_{\mathbf{q}=\lambda \mathbf{k}_{\gamma}}$

the magnetic moment $\mathbf{M}_{\text {int }}\left(\mathbf{k}_{\gamma}\right)=\mathbf{M}_{\text {int }}^{\text {orb }}\left(\mathbf{k}_{\gamma}\right)+\mathbf{M}_{\text {int }}^{\text {spin }}\left(\mathbf{k}_{\gamma}\right)+\mathbf{M}_{\text {int }}^{[2]}\left(\mathbf{k}_{\gamma}\right)$,

where $f_{\alpha}^{N}(\mathbf{q})$ is the nucleon form factor (FF).

\section{Model calculations in a simple cluster picture}

Now, we will consider the following reaction $\alpha+\alpha \rightarrow \alpha+\alpha+\gamma$ with the internal Hamiltonian

$$
H_{\text {int }}=H_{1}^{\text {int }}+H_{2}^{\text {int }}+\frac{\left(\mathbf{P}_{1}-\mathbf{P}_{2}\right)^{2}}{16 m}+V
$$

where $\mathbf{P}_{1}, \mathbf{P}_{2}$ - the total momentum operators, $m$ - nucleon mass and $H_{1,2}^{\text {int }}$ the exact "microscopic" Hamiltonians

$$
H_{1,2}^{i n t} \equiv H_{1,2}-\frac{\mathbf{P}_{1,2}^{2}}{8 m}, H_{1,2}=\sum_{i \in \alpha_{1,2}} \frac{\mathbf{p}_{i}^{2}}{2 m}+\sum_{i<k} V(i, k),
$$

of the colliding alpha particles and the interaction $V$ between them, $V=\sum_{i \in \alpha_{1}, k \in \alpha_{2}} V(i, k)$.

An essential simplification of subsequent calculations is achieved via 1) replacing the exact interaction $V$ in $H_{\text {int }}$ by an effective one $V_{\text {eff }}\left(\alpha_{1}, \alpha_{2}\right)$ which depends on the cluster-cluster relative coordinate $\mathbf{r}=\mathbf{R}_{1}-\mathbf{R}_{2}$, where $\mathbf{R}_{1,2}$ the corresponding CM coordinates and 2) assuming that the $H_{\text {int }}$ eigenvectors are approximated schematically to

$$
|i\rangle=\Phi_{\alpha_{1}} \Phi_{\alpha_{2}} \chi^{(+)},|f\rangle=\Phi_{\alpha_{1}^{\prime}} \Phi_{\alpha_{2}^{\prime}} \chi^{(-)},
$$

with the "distorted" waves $\chi_{\mathbf{k}}^{( \pm)}$that describe the cluster-cluster scattering

$$
\left[\frac{\mathbf{k}^{2}}{2 \mu_{\alpha}}+V_{e f f}\left(\alpha_{1}, \alpha_{2}\right)\right]\left|\chi_{\mathbf{k}}^{( \pm)}\right\rangle \equiv H_{r e l}\left|\chi_{\mathbf{k}}^{( \pm)}\right\rangle=E_{r e l}\left|\chi_{\mathbf{k}}^{( \pm)}\right\rangle
$$

Moreover, we will deal with the ${ }^{4} H e$ g.s.: $\Phi_{\alpha_{1}}=\Phi_{\alpha_{2}}=\Phi_{\alpha_{1}^{\prime}}=\Phi_{\alpha_{2}^{\prime}}=\Phi_{\alpha}$.

Here we will confine ourselves only to the first term in Eq.(8). Doing so we have in the Coulomb gauge

$$
T_{i f}=-\frac{1}{\sqrt{2(2 \pi)^{9} E_{\gamma}}} \boldsymbol{\varepsilon}\left(\mathbf{k}_{\gamma}\right) \cdot \mathbf{m}\left(\mathbf{k}_{\gamma}\right)
$$


with

$$
\mathbf{m}\left(\mathbf{k}_{\gamma}\right)=E_{\gamma} \int_{0}^{1}\left[\nabla_{\mathbf{q}}+\frac{\mathbf{P}_{i}}{2 m_{\alpha} E_{\gamma}}\right] F_{C H}(q)\left(I\left(\mathbf{k}^{\prime}, \mathbf{k} ; \mathbf{q}\right)+I\left(\mathbf{k}^{\prime}, \mathbf{k} ;-\mathbf{q}\right)\right) d \lambda,
$$

where $F_{C H}(q)$ is the charge FF of the alpha particle [7] and $I\left(\mathbf{k}^{\prime}, \mathbf{k} ; \mathbf{q}\right)$ is the overlap integral

$$
I\left(\mathbf{k}^{\prime}, \mathbf{k} ; \mathbf{q}\right)=\left\langle\chi_{\mathbf{k}^{\prime}}^{(-)}\left|e^{-i \frac{1}{2} \mathbf{q r}}\right| \chi_{\mathbf{k}}^{(+)}\right\rangle=\int d \mathbf{r} \chi_{\mathbf{k}^{\prime}}^{(-) *}(\mathbf{r}) e^{-i \frac{1}{2} \mathbf{q r}} \chi_{\mathbf{k}}^{(+)}(\mathbf{r}),
$$

with the stretched photon momentum $\mathbf{q}=\lambda \mathbf{k}_{\gamma}$.

\section{The initial and final state interactions included}

In many cases typical of the nuclear physics the distorted waves in Eq.(14) should be evaluated nonperturbatively. Therefore, we prefer to go on addressing the so-called two-potential problem ( see, e.g., [8]). First, we separate out the point-like Coulomb interaction

$$
V=V_{C}+\left(V-V_{C}\right) \equiv V_{C}+V_{C S}, \quad V_{C}=4 e^{2} / r
$$

that allow us to use the following decomposition of the total resolvent

$$
G(z)=(z-H)^{-1}, \quad G(z)=G_{C}(z)+G_{C}(z) V_{C S} G(z),
$$

with the Coulomb resolvent $G_{C}(z)=\left(z-H_{C}\right)^{-1}, H_{C}=H_{0}+V_{C}$. Such a decomposition gives

$$
\left|\chi_{\mathbf{k}}^{( \pm)}\right\rangle= \pm i \lim _{\epsilon \rightarrow 0+} \epsilon G\left(E_{k} \pm i \epsilon\right)|\mathbf{k}\rangle \rightarrow\left|\chi_{\mathbf{k}}^{( \pm)}\right\rangle=\left|\psi_{C \mathbf{k}}^{( \pm)}\right\rangle+G_{C}\left(E_{\mathbf{k}} \pm i 0\right) V_{C S}\left|\chi_{\mathbf{k}}^{( \pm)}\right\rangle \text {, }
$$

with the Coulomb scattering wave function $\left|\psi_{C \mathbf{k}}^{( \pm)}\right\rangle$. Further, for the repulsive Coulomb potential we employ

$$
G_{C}\left(E_{k} \pm i 0\right)=\int \frac{\left|\psi_{C \mathbf{p}}^{( \pm)}\right\rangle d \mathbf{p}\left\langle\psi_{C \mathbf{p}}^{( \pm)}\right|}{E_{k} \pm i 0-E_{p}} \rightarrow \chi_{\mathbf{k}}^{(+)}(\mathbf{r})=\psi_{C \mathbf{k}}^{(+)}(\mathbf{r})+\int d \mathbf{q} \frac{\psi_{C \mathbf{q}}^{(+)}(\mathbf{r})\left\langle\psi_{C \mathbf{q}}^{(+)}\left|V_{S}\right| \chi_{\mathbf{k}}^{(+)}\right\rangle}{E_{k}+i 0-E_{q}},
$$

which leads to

$$
\left|\chi_{\mathbf{k}}^{( \pm)}\right\rangle=\left|\psi_{C \mathbf{k}}^{( \pm)}\right\rangle+G_{C}\left(E_{\mathbf{k}} \pm i 0\right) V_{C S}\left|\chi_{\mathbf{k}}^{( \pm)}\right\rangle .
$$

In its turn, overlap integral (17) splits into two parts (cf. decomposition Eq. (1) in [10])

$$
I\left(\mathbf{k}^{\prime}, \mathbf{k} ; \mathbf{q}\right)=\left\langle\chi_{\mathbf{k}^{\prime}}^{(-)}\left|e^{-i \frac{1}{2} \mathbf{q r}}\right| \chi_{\mathbf{k}^{\prime}}^{(+)}\right\rangle=I_{C}+I_{C S}, I_{C S} \equiv I-I_{C},
$$

with the Nordsieck-type integral related to purely Coulomb contribution

$$
I_{C}\left(\mathbf{k}^{\prime}, \mathbf{k} ; \mathbf{q}\right)=\left\langle\psi_{c \mathbf{k}^{\prime}}^{(-)}\left|e^{-i \frac{1}{2} \mathbf{q} \hat{\mathbf{r}}}\right| \psi_{c \mathbf{k}}^{(+)}\right\rangle
$$

and mixed Coulomb-strong interaction integral

$$
\begin{gathered}
I_{C S}\left(\mathbf{k}^{\prime}, \mathbf{k} ; \mathbf{q}\right)=\left\langle\chi_{c \mathbf{k}^{\prime}}^{(-)}\left|V_{C S} G_{C}\left(E_{k}+i 0\right) e^{-i \frac{1}{2} \mathbf{q} \hat{\mathbf{r}}}\right| \chi_{c \mathbf{k}}^{(+)}\right\rangle+\left\langle\chi_{c \mathbf{k}^{\prime}}^{(-)}\left|e^{-i \frac{1}{2} \mathbf{q} \hat{\mathbf{r}}} G_{C}\left(E_{k}+i 0\right) V_{C S}\right| \chi_{c \mathbf{k}}^{(+)}\right\rangle+ \\
\left\langle\chi_{c \mathbf{k}^{\prime}}^{(-)}\left|V_{C S} G_{C}\left(E_{k}+i 0\right) e^{-i \frac{1}{2} \mathbf{q} \hat{\mathbf{r}}} G_{C}\left(E_{k}+i 0\right) V_{C S}\right| \chi_{c \mathbf{k}}^{(+)}\right\rangle .
\end{gathered}
$$

Analytical expression for the integral $I_{C}$ can be found in [11] (see Eq. (10) therein). Calculations of the three dimenslional integral $I_{C S}$ are reduced to the summation of its partial wave expansions with radial integrals

$$
I_{C S}\left(l^{\prime}, l, L\right)=\int_{0}^{\infty} d r j_{L}\left(\frac{1}{2} q r\right)\left[g_{l^{\prime} l} w_{k^{\prime} l^{\prime}}(r) w_{k l}(r)+w_{k^{\prime} l^{\prime}}(r) w_{k l}(r)-F_{l^{\prime}}\left(k^{\prime} r\right) F_{l}(k r)\right],
$$


with $w_{k l}(r)=(k r) \psi_{k, l}(r)$ and quantities $g_{l^{\prime} l}=\frac{1}{4}\left(s_{l^{\prime}}-1\right)\left(s_{l}-1\right)+\frac{1}{2}\left(s_{l^{\prime}}-1\right)+\frac{1}{2}\left(s_{l}-1\right)$ being expressed in terms of the $S$-matrix elements in the angular momentum representation $s_{l}=e^{2 i \delta_{l}(k)}$, where $\delta_{l}(k)$ are phase shifts for given values of the collision energy and angular momentum $l$.

The convergence of the $I_{C S}$ partial expansion provided owing to that quantities $g_{l^{\prime} l}$ vanish at large $l$ and $l^{\prime}$, since $\left|s_{l}-1\right| \rightarrow 0$ and wave function $w_{k l}(r)$ coincides with the Coulomb function $F_{l}(k r)$ at large $l$. At intermediate energies $(E \sim 10 \mathrm{MeV})$ one can confine ourselves to $l_{\max }^{\prime}=l_{\max }=6$. In order to evaluate radial integrals (26) it is convenient to employ the the contour integration method [12] (see also its comparison to other ones in [13]).

\section{Results and discussion}

One of motivations in studying the $\alpha+\alpha \rightarrow \alpha+\alpha+\gamma$ bremsstrahlung is to get a supplementary information on a strong part of the alpha-alpha interaction. In the context, we will consider some correlation function $d \sigma \equiv d^{5} \sigma / d E_{\gamma} d \Omega_{1 i} d \Omega_{1 f}$, in which one of the outgoing alphas is detected in coincidence with the emitted photon

$$
d \sigma=\frac{(2 \pi)^{10} E_{\gamma}^{2} M_{\alpha}^{2}}{k_{1 i} \sqrt{\left(k_{1 i} \cos \left(\theta_{1 i}-\theta_{1 f}\right)-E_{\gamma} \cos \theta_{1 f}\right)^{2}-4 M_{\alpha} E_{\gamma}}}\left[k_{1 f}^{(+) 2}\left|T_{i f}\left(k_{1 f}^{(+)}\right)\right|^{2}+k_{1 f}^{(-) 2}\left|T_{i f}\left(k_{1 f}^{(-)}\right)\right|^{2}\right],
$$

where $k_{1 i}=\sqrt{2 M_{\alpha} E_{i}}$ momentum of the initial $\alpha$-particle, $E_{\gamma}$ energy of the emitted photon, $M_{\alpha}$ the $\alpha$-particle mass. One of the outgoing alphas has the momentum fixed by the total momentum conservation $\mathbf{k}_{2 f}=\mathbf{k}_{1 i}-\mathbf{k}_{1 f}-\mathbf{k}_{\gamma}$. The other one with momentum $\mathbf{k}_{1 f}$ is detected in coincidence with the emitted photon and its kinematically permissible values are given by $k_{1 f}^{( \pm)}$. All momenta have a coplanar disposal, where the photon with its momentum is directed along the Z-axis and the rest momenta lie in the XZ-plane, viz., $\hat{\mathbf{k}}_{1}=\left(\theta_{1 i}, 0\right), \hat{\mathbf{k}_{1 f}}=\left(\theta_{1 f}, \pi\right)$. As

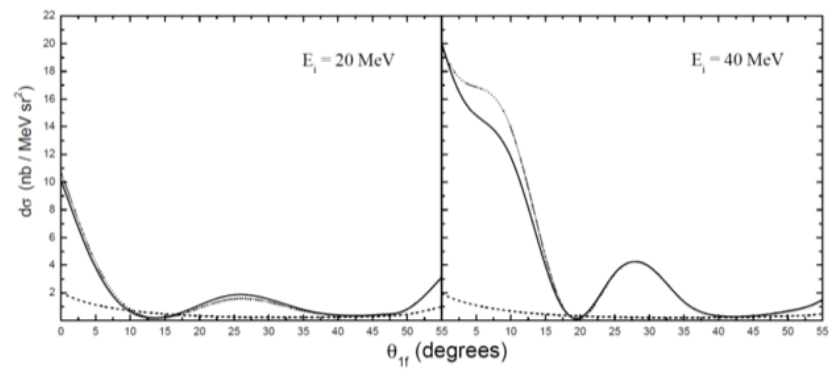

Figure 1: Bremsstrahlung cross section for different values of incident energies $E_{i}$ and for photon energy $E_{\gamma}=1 \mathrm{MeV}$ : calculated for potential by Buck et al. [14] (solid curve) and potential by Ali Bodmer [15] (dotted). The pure Coulomb contribution is shown by the dashed curves.

seen in Fig.1, when increasing the incident energy the cross-section becomes more sensitive to the choice the model $\alpha-\alpha$ interaction. By relying upon this observation it would be desirable to measure such cross sections at medium $\alpha-\alpha$ collision energies feasible with many available accelerators. It would allow to make up a scarce information on the interaction between alpha particles at short distances. 


\section{Acknowledgements}

One of us A.A is very thankful to the Organizing Committee of the 24th European Conference on Few-Body Problems in Physics and the International Relations Office of V. N. Karazin Kharkiv National University for the financial assistance of his participation in this conference.

\section{References}

[1] A. V. Shebeko, Towards gauge-independent treatment of radiative capture in nuclear reactions: Applications to low-energy cluster-cluster collisions, Phys. Atom. Nuclei 77, 518 (2014), doi:10.1134/S1063778814040139.

[2] A. Shebeko, A generalization of Siegert's theorem and separation of center-of-mass motion, Sov. J. Nucl. Phys. 49, 30 (1989).

[3] L. Levchuk and A. Shebeko, On a generalization of Siegert's theorem. A corrected result, Phys. At. Nucl. 56, 227 (1993).

[4] J. L. Friar and S. Fallieros, Gauge-invariant nuclear Compton amplitude manifesting lowenergy theorems, Phys. Rev. C 34, 2029 (1986), doi:10.1103/PhysRevC.34.2029.

[5] J. L. Friar and W. C. Haxton, Current conservation and the transverse electric multipole field, Phys. Rev. C 31, 2027 (1985), doi:10.1103/PhysRevC.31.2027.

[6] L. Levchuk, L. Canton and A. Shebeko, Nuclear effects in positive pion electroproduction on the deuteron near threshold, Eur. Phys. J. A 21, 29 (2004), doi:10.1140/epja/i200310184-1.

[7] A. V. Shebeko, P. A. Grigorov and V. S. Iurasov, Translationally invariant calculations of form factors, nucleon densities and momentum distributions for finite nuclei with short-range correlations included, Eur. Phys. J. A 48, 153 (2012), doi:10.1140/epja/i2012-12153-y.

[8] C. Elster, L. C. Liu and R. M. Thaler, A practical calculational method for treating Coulomb scattering in momentum space, J. Phys. G: Nucl. Part. Phys 19, 2123 (1993), doi:10.1088/0954-3899/19/12/015.

[9] G.E Brown and A.D. Jackson, Nucleon-nucleon interaction, North-Holland Publ. Co., Amsterdam (1976).

[10] D. Baye, C. Sauwens, P. Descouvemont and S. Keller, Accurate treatment of Coulomb contribution in nucleus-nucleus bremsstrahlung, Nucl. Phys. A 529, 467 (1991), doi:10.1016/0375-9474(91)90581-P.

[11] M. S. Gravielle and J. E. Miraglia, Some Nordsieck integrals of interest in radiation and atomic collision theories, Comput. Phys. Commun. 69, 53 (1992), doi:10.1016/00104655(92)90127-K.

[12] C. M. Vincent and H. T. Fortune, New method for distorted-wave analysis of stripping to unbound states, Phys. Rev. C 2, 782 (1970), doi:10.1103/PhysRevC.2.782.

[13] J. Dohet-Eraly and D. Baye, Comparison of potential models of nucleus-nucleus bremsstrahlung, Phys. Rev. C 90, 034611 (2014), doi:10.1103/PhysRevC.90.034611. 
[14] B. Buck, H. Friedrich and C. Wheatley, Local potential models for the scattering of complex nuclei, Nucl. Phys. A 275, 246 (1977), doi:10.1016/0375-9474(77)90287-1.

[15] S. Ali and A. R. Bodmer, Phenomenological $\alpha$ - $\alpha$ potentials, Nucl. Phys. 80, 99 (1966), doi:10.1016/0029-5582(66)90829-7. 